\title{
Expression of Rab1A in bladder cancer and its clinical implications
}

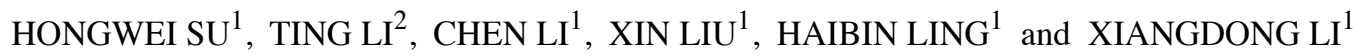 \\ ${ }^{1}$ Department of Urology, The First Affiliated Hospital of Hebei North University; \\ ${ }^{2}$ Department of Drug and Equipment, The Second Hospital of Zhangjiakou City, Zhangjiakou, Hebei 075061, P.R. China
}

Received September 8, 2018; Accepted April 11, 2019

DOI: $10.3892 /$ etm.2020.9174

\begin{abstract}
Rab1A protein has been identified to be highly expressed in a number of malignant tumor tissues and to participate in the regulation of tumor development, but no data concerning bladder cancer have been described at present. The present study measured the expression of Rab1A in bladder cancer tissues and cell lines, and analyzed its clinical significance for patients with bladder cancer. A total of 153 pairs of bladder cancer tumor tissues and adjacent cancer healthy tissues were included in the present study. Western blot analysis and immunohistochemistry were used to measure the expression of Rab1A protein in normal bladder and bladder cancer cell line, and bladder cancer and normal adjacent tissues. SPSS 20.0 software was used for statistical analysis and mapping of survival curves in patients with bladder cancer. The expression levels of Rab1A protein in normal bladder cells and tissues was significantly decreased compared with that in bladder cancer cells and tissues, and it was significantly associated with tumor size, histological grade, tumor-node-metastasis (TNM) stage, lymph node metastasis and remote metastasis in 153 patients with bladder cancer. Cox regression analysis demonstrated that the expression of Rab1A protein in bladder cancer tissues was an independent risk factor for prognosis (overall risk $=0.549 ; 95 \%$ confidence interval $=0.139-0.916)$. The 5 -year survival rate of patients with bladder cancer with high expression levels of Rab1A protein was $48.613 \%$, which was significantly decreased compared with the rate of patients with low expression $75.31 \%(\mathrm{P}<0.05)$. The expression of Rab1A in bladder cancer tissues and cell lines was upregulated, and its expression increased with increasing TNM stages. It was also associated with the metastasis of tumor cells and negatively affected the survival time of patients with bladder cancer.
\end{abstract}

Correspondence to: Dr Hongwei Su, Department of Urology, The First Affiliated Hospital of Hebei North University, 12 Changqing Road, Qiaoxi, Zhangjiakou, Hebei 075061, P.R. China

E-mail: suhwwww@163.com

Key words: RAB1A, member RAS oncogene family, bladder cancer, clinical implications

\section{Introduction}

Bladder cancer is one of the most common malignant tumors in the urogenital system. The incidence of bladder cancer currently ranks 11th among all malignant tumors and $\sim 15,000$ people succumb to bladder cancer each year worldwide (1). In China, bladder cancer is the genitourinary tumor with the highest morbidity and mortality. The incidence rate in males is $\sim 7.3 / 100,000$, and in females it is $\sim 2.0 / 100,000$. The incidence of bladder cancer in China is also increasing year by year $(2,3)$. Consequently, bladder cancer has attracted increased attention due to its high incidence and recurrence rates, which threaten human health.

The human RAB1A, member RAS oncogene family (Rab1A) gene is located at chromosome 2 q14 and is primarily expressed in the endoplasmic reticulum and Golgi apparatus (4); it is known to be involved in vesicle trafficking between the endoplasmic reticulum and the Golgi apparatus $(5,6)$. Rab1A, a member of the Ras-associated binding (Rab) family, is a small guanosine triphosphate (GTP) enzyme that is an activator of mammalian rapamycin target protein complex 1 (mTORC1) $(7,8)$, and has been demonstrated to be involved in the regulation of vesicle transport from the endoplasmic reticulum to the Golgi $(9,10)$. A number of previous studies identified that Rab1A protein was also involved in the regulation of signal transduction (11), cell migration (12) and autophagy $(13,14)$. Concomitantly, the abnormal expression of Rab1A was also associated with the development of certain clinical diseases, including Parkinson's disease (15) and primary cardiomyopathy (16). In recent years, studies have begun to examine to the role of Rab1A in tumorigenesis and development, and have identified that Rab1 A was increased in a number of malignant tumors and served an important role in the development of tumors, including tongue (17), breast (18), lung (19), liver (20) and colorectal cancer (21,22). However, the expression pattern of Rab1A in bladder cancer remains unknown.

In the present study, the expression levels of Rab1A protein in the cells and tissues of bladder cancer were measured, and its clinical significance for patients with bladder cancer was analyzed. It was identified that Rab1A protein was highly expressed in bladder cancer cells and tissues, and was associated with the development of bladder cancer, and that it may be a potential target for the treatment of bladder cancer. 


\section{Materials and methods}

Study objects and clinical specimens. A total of 153 patients with bladder cancer (121 males and 32 females) were selected as the patient cohort from January 2011 to December 2012 in The First Affiliated Hospital of Hebei North University (Zhangjiakou, China). The cancer tissues and adjacent cancer normal tissues were collected following surgical resection. Each tissue sample was divided into 3 sections; 1 was stored as paraffin sections for use in the immunohistochemistry (IHC) analysis, and 2 sections were stored in liquid nitrogen. The age range of the 153 patients with bladder cancer was 45-72 years (median age, 65 years); additional clinical data is summarized in Table I. Exclusion criteria were as follows: i) Incomplete age, sex, disease history and tumor information; ii) postoperative follow-up loss or unknown cause of mortality; iii) presence of other malignancies, or malignancies that could not be identified as primary liver cancer due to mortality from other sudden diseases (including cardiovascular and cerebrovascular diseases), or evidence of poor postoperative mental status affecting patient prognosis; iv) patients who were pregnant or breast-feeding; and v) patients with chronic infectious diseases (human immunodeficiency virus, hepatitis B or tuberculosis).

In addition, all subjects (or their guardians) included in the present study consented to the study protocol and provided written informed consent. The Ethics Committee of The First Affiliated Hospital of Hebei North University approved the study.

Cell and cell culture. The normal human bladder epithelial SV-HUC-1 cell line, and human bladder cancer ScaBER (HTB-3), 5637 (HTB-9) and T24 (HTB-4) cell lines were obtained from the American Type Culture Collection and were cultured in Dulbecco's modified Eagle medium (Thermo Fisher Scientific, Inc.), to which $10 \%$ fetal bovine serum (Thermo Fisher Scientific, Inc.) and 1\% penicillin-streptomycin (Thermo Fisher Scientific, Inc.) were added.

Western blot analysis. Firstly, RIPA buffer (Beijing Solarbio Science \& Technology Co., Ltd.) was used to exact total protein from tissues and cells, where a Bicinchoninic Acid Assay kit (Beyotime Institute of Biotechnology) was used to quantify protein concentration. A total of $40 \mu \mathrm{g}$ protein was subsequently separated by $10 \%$ SDS-PAGE and transferred to polyvinylidene fluoride membranes. The membranes were blocked using 5\% bovine serum albumin (Sigma-Aldrich; Merck $\mathrm{KGaA}$ ) solution for $1 \mathrm{~h}$ at room temperature, and block solution was used to dilute primary and secondary antibodies. The primary antibodies used were as follows: Anti-Rab1A (cat. no., ab97956; 1:1,000; Abcam) or anti-GAPDH (cat. no., ab9484; 1:3,000; Abcam). The secondary antibodies were as follows: Horseradish peroxidase (HRP)-conjugated goat anti-Rabbit IgG H\&L (cat. no. ab205718; 1:30,000; Abcam) or horseradish peroxidase-conjugated goat anti-mouse (cat. no., ab6789; 1:3,000; Abcam). The membranes were incubated with the primary antibodies overnight at $4^{\circ} \mathrm{C}$, followed by incubation with corresponding second antibodies for $1 \mathrm{~h}$ at room temperature. Western Lightening ${ }^{\mathrm{TM}}$ Plus-ECL (PerkinElmer, Inc.) was used to visualize the protein bands,
Table I. Association between Rab1A expression and clinical data of patients with bladder cancer.

\begin{tabular}{|c|c|c|c|c|c|}
\hline \multirow[b]{2}{*}{ Characteristics } & \multirow[b]{2}{*}{$\mathrm{N}$} & \multicolumn{2}{|c|}{$\begin{array}{c}\text { Rab1A } \\
\text { expression }\end{array}$} & \multirow[b]{2}{*}{$\chi^{2}$} & \multirow[b]{2}{*}{ P-value } \\
\hline & & Low & High & & \\
\hline \multicolumn{6}{|l|}{ Sex } \\
\hline Female & 32 & 18 & 14 & 0.178 & 0.673 \\
\hline Male & 121 & 63 & 58 & & \\
\hline \multicolumn{6}{|l|}{ Age, years } \\
\hline$<60$ & 62 & 32 & 30 & 0.114 & 0.736 \\
\hline$\geq 60$ & 91 & 49 & 42 & & \\
\hline \multicolumn{6}{|l|}{ Tumor size, $\mathrm{cm}$} \\
\hline$<2.5$ & 81 & 51 & 30 & 6.149 & 0.013 \\
\hline$\geq 2.5$ & 72 & 30 & 42 & & \\
\hline \multicolumn{6}{|l|}{ Histological grade } \\
\hline I & 72 & 42 & 30 & 15.282 & $<0.001$ \\
\hline II & 43 & 29 & 14 & & \\
\hline III & 38 & 10 & 28 & & \\
\hline \multicolumn{6}{|l|}{ Tumor number } \\
\hline Single & 92 & 49 & 43 & 0.009 & 0.922 \\
\hline Multiple & 61 & 32 & 29 & & \\
\hline \multicolumn{6}{|l|}{ TNM } \\
\hline 0a-II & 85 & 66 & 19 & 48.856 & $<0.001$ \\
\hline III-IV & 68 & 15 & 53 & & \\
\hline \multicolumn{6}{|l|}{ Lymph node metastasis } \\
\hline Yes & 28 & 8 & 20 & 8.170 & 0.004 \\
\hline No & 125 & 73 & 52 & & \\
\hline \multicolumn{6}{|l|}{ Remote metastasis } \\
\hline Yes & 10 & 0 & 10 & 12.037 & 0.001 \\
\hline No & 143 & 81 & 62 & & \\
\hline \multicolumn{6}{|l|}{ Tumor category } \\
\hline Urothelium & 136 & 70 & 66 & 1.063 & 0.303 \\
\hline Squamous cell carcinoma & 17 & 11 & 6 & & \\
\hline
\end{tabular}

Rab1A, RAB1A, member RAS oncogene family.

and Image $\mathbf{J}$ version 1.50d (National Institutes of Health) was used to perform densitometric analysis.

IHC. Paraffin-embedded sections of preserved bladder cancer tumor and adjacent cancer tissues were selected, and IHC was used to detect the expression of Rab1A protein. All experimental protocols were performed according to the protocols of VECTASTAIN ${ }^{\circledR}$ Elite $^{\circledR}$ ABC kit (Vector Laboratories, Inc.) and analyzed using a Leica TCS SP5 light microscope (Leica Microsystems, Inc.) using the LAS AF Lite image browser software (version 4.0; Leica Microsystems, Inc.). Anti-Rab1 A (cat. no., ab97956; 1:100; Abcam) was selected as the primary antibody and the horseradish-conjugated goat anti-rabbit IgG (H\&L) (cat. no., ab6702; 1:2,000; Abcam) was selected as the secondary antibody. Sections were 

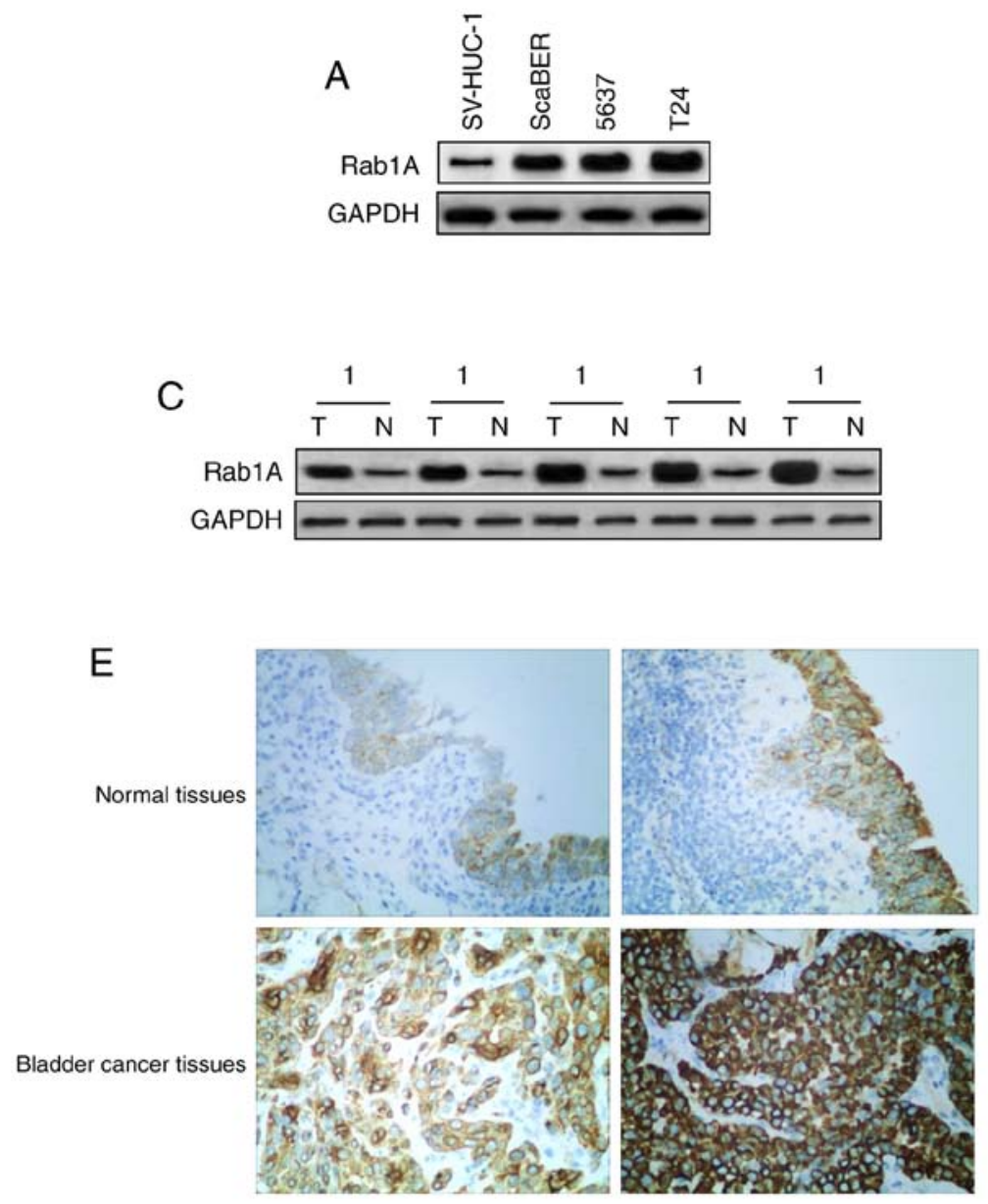
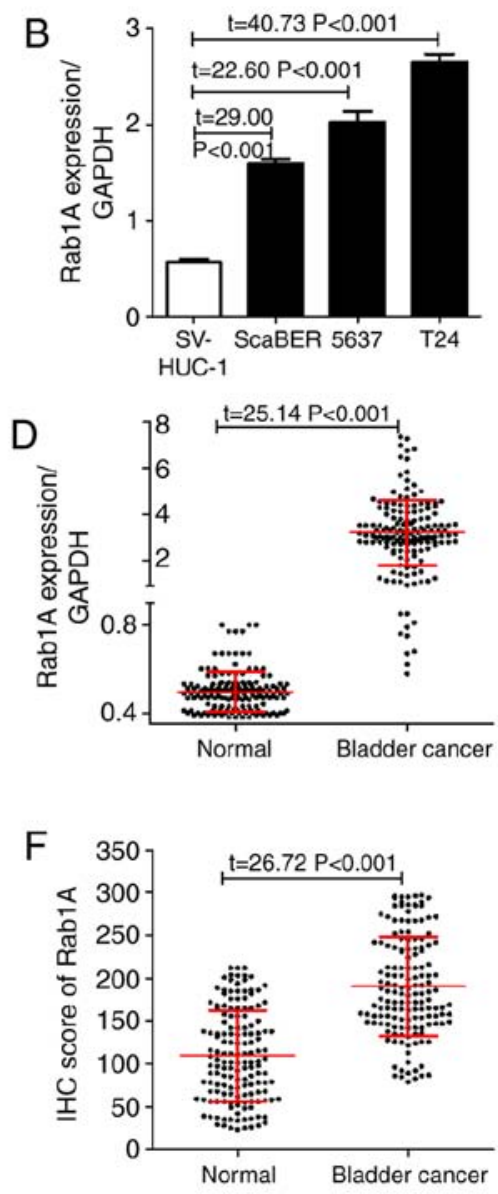

Figure 1. Upregulation of Rab1A protein expression in bladder cancer tissues and cells. (A) Representative western blot analysis gel measuring the protein expression levels of Rab1A protein in the normal human bladder epithelial SV-HUC-A cell line and human bladder cancer ScaBER, 5637 and T24 cell lines. (B) Densitometric analysis of the relative western blot analysis gray values. (C) Representative western blot analysis gel measuring the protein expression levels of Rab1A protein in human bladder cancer and the adjacent normal tissues. (D) Densitometric analysis of the relative western blot analysis gray values. (E and F) IHC was used to measure the expression of Rab1A protein in human bladder and the adjacent normal tissues. (E) Representative tissue samples. Magnification, x100. (F) IHC scores. Rab1A, RAB1A, member RAS oncogene family; IHC, immunohistochemistry.

incubated with the primary antibody overnight at $4^{\circ} \mathrm{C}$ and with the secondary antibody for $2 \mathrm{~h}$ at $37^{\circ} \mathrm{C}$. The scoring method used from five sections per condition in the present study as described previously by Shimada et al (17), which was calculated based on the staining intensity and percentage of stained cells: 0 , No appreciable staining in cells; 1 , weak staining in cells comparable to stromal cells; 2 , intermediate staining; 3 , strong staining. The fraction of positive cells was scored as $0-100 \%$. The IHC score was calculated by multiplying the intensity and the fraction scores, producing a total range of $0-300$.

Statistical analysis. SPSS 20.0 software (IBM Corp.) was used to perform the statistical analysis on the data. Measurement data are presented as the mean \pm standard deviation. Differences between two groups was compared by a paired Student's t-test for paired data, and data from multiple groups was analyzed using a one-way analysis of variance followed by Duncan's post hoc test. Counts were recorded as proportions, and the differences between groups were compared using a $\chi^{2}$ test. Kaplan-Meier curves were used to analyze the survival data, and a log-rank test was used to compare the survival of patients with high and low Rab1 A expression levels. A Cox regression model was used to analyze factors that affected the survival of patients with bladder cancer. $\mathrm{P}<0.05$ was considered to indicate a statistically significant difference.

\section{Results}

RablA is highly expressed in human bladder cancer tissues and cells. As demonstrated in Fig. 1A and B, it was identified that the expression of Rab1A protein in the normal human bladder epithelial SV-HUC-1 cell line was significantly decreased compared with that in the human bladder cancer ScaBER, 5637 and T24 cell lines. In addition, the Rab1 A expression levels in 153 pairs of bladder cancer tissues and adjacent normal tissues were detected by western blot analysis. The results revealed that the relative expression of RablA in tumor tissues was $3.20 \pm 1.42$, which was significantly increased compared with that in the adjacent normal tissues $(0.50 \pm 0.09)$ $(\mathrm{P}<0.05$; Fig. $1 \mathrm{C}$ and $\mathrm{D})$.

In addition, IHC was also used to measure the expression of RablA in tissues and determine the patterns of distribution in bladder cancer cells; it was identified that the IHC score of 

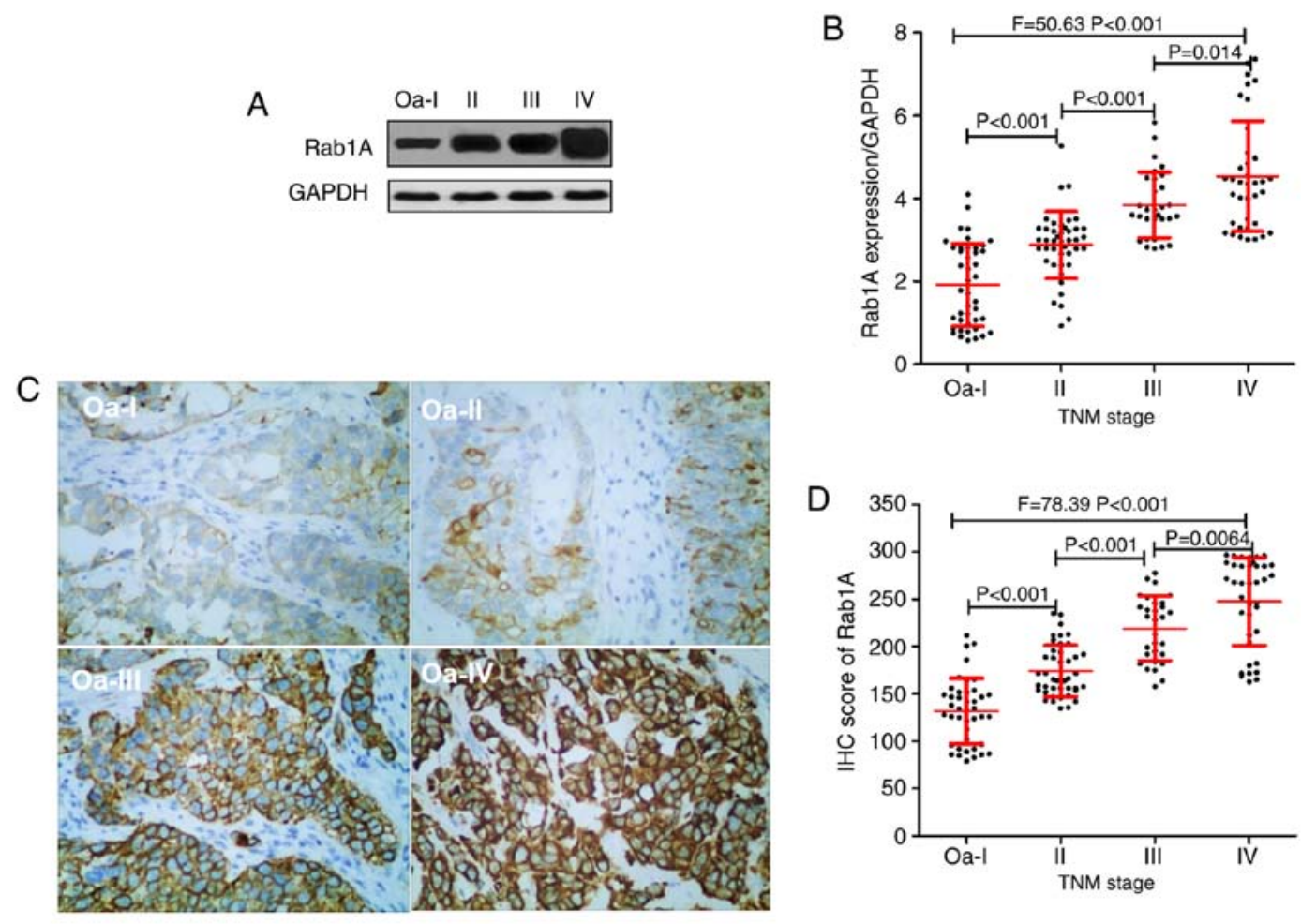

Figure 2. Rab1A protein increases with increasing TNM grade. (A and B) Western blot analysis was used to measure the expression of Rab1A protein in difference TNM stage. (A) Representative western blot analysis gel. (B) Densitometric analysis of the relative western blot analysis gray values. (C and D) IHC was used to measure the expression of Rab1 A protein in different TNM stages. (C) Representative tissue samples. Magnification, x100. (D) IHC scores. Rab1A, RAB1A, member RAS oncogene family; TNM, tumor-node-metastasis; IHC, immunohistochemistry.
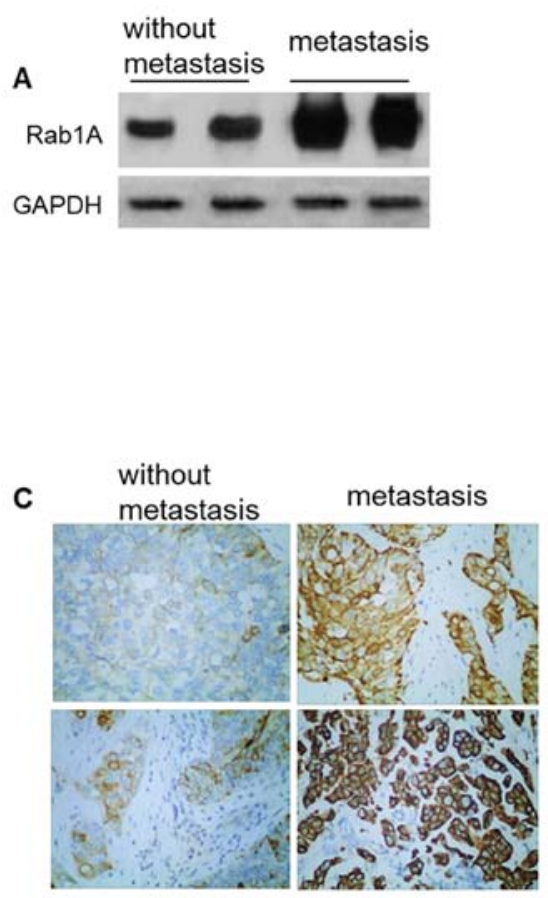
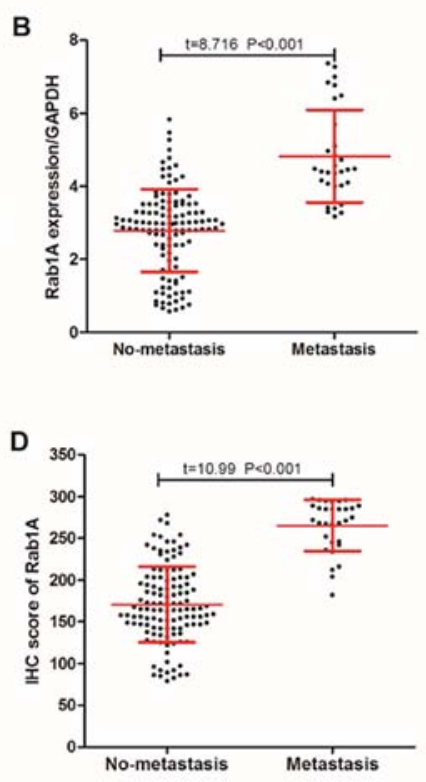

Figure 3. Rab1 A protein expression promotes bladder cancer cell metastasis. (A) Representative western blot analysis gel of Rab1A protein expression levels in tumor tissues with or without metastasis. (B) Densitometric analysis of the relative western blot analysis gray values. (C and D) Immunohistochemistry was used to measure the expression of Rab1A protein in tumor tissue with or without metastasis. (C) Representative tissue samples. Magnification, x100. (D) IHC scores. Rab1A, RAB1A, member RAS oncogene family; IHC, immunohistochemistry.

the 153 bladder cancer tissues was $190.65 \pm 57.77$, which was significantly increased compared with that in the adjacent normal tissues $(109.57 \pm 53.12)(\mathrm{P}<0.05$; Fig. $1 \mathrm{E}$ and $\mathrm{F})$.
Association between RablA and clinical data of patients with bladder cancer. A total of 153 patients with bladder cancer were divided into two groups depending on the expression of Rab1 A 
protein in cancer tissues, which was measured by western blot analysis, using the median value as the cut-off: Low Rab1A expression was identified in 81 cases $[<3.17$ (median) of 153 patients with bladder cancer] and 72 cases exhibited high Rab1A expression $[\geq 3.17$ (median) of 153 patients with bladder cancer]. In addition, the association between Rab1A expression and clinicopathological features in patients with bladder cancer was analyzed. As demonstrated in Table I, the expression of Rab1A was not associated with age, sex, tumor number and tumor category, but was significantly associated with tumor size, histological grade, tumor-node-metastasis (TNM) stage (2002 version) $(23,24)$, lymph node metastasis and remote metastasis.

RablA protein expression and TNM stage of bladder cancer. The TNM stages of 153 patients with bladder were assessed; 42, 43, 30 and 38 patients were diagnosed with grade $0 \mathrm{a}-\mathrm{I}$, II, III and IV, respectively. The results of the western blot analysis suggested that the expression of Rab1A protein in 0a-I, II, III, and IV bladder cancer tissues were $1.92 \pm 1.00,2.88 \pm 0.81$, $3.85 \pm 0.79$ and $4.54 \pm 1.33$, respectively (Fig. 2A and B). IHC analysis indicated that the Rab1 A protein IHC scores were $131.86 \pm 34.47,174.37 \pm 27.16,219.23 \pm 34.06$ and $247.74 \pm 46.44$ in the grade 0a-I, II, III and IV bladder cancer tissues, respectively (Fig. 2C and D).

RablA protein expression and bladder cancer cell metastasis. A total of 31 patients with bladder cancer were identified to exhibited cancer cell metastasis (lymph node metastasis and distant metastasis), and the expression of RablA protein in tumor tissues of patients with metastatic and non-metastatic bladder cancer were compared. The results of the western blot analysis demonstrated that the relative expression of Rab1A protein was $(4.82 \pm 1.27)$ in the metastatic bladder cancer tissues, which was significantly $(\mathrm{P}<0.001)$ increased compared with that in the non-metastatic bladder cancer tissues $(2.79 \pm 1.13$; Fig. 3A and B).

In addition, IHC data suggested that (Fig. 3C and D) the Rab1A protein IHC score was $265.16 \pm 30.83$ in the metastatic bladder cancer tissues, which was significantly $(\mathrm{P}<0.001)$ increased compared with that in the non-metastatic bladder cancer tissues $(170.54 \pm 40.28)$.

Effect of RablA protein on the prognosis of patients with bladder cancer. The results of the univariate and multivariate Cox regression models indicated that Rab1A was an independent risk factor for survival in patients with bladder cancer (odds ratio $=0.549 ; 95 \%$ confidence interval $=0.139-0.916)$ (Tables II and III). In addition, a 5-year follow-up of the 153 patients with bladder cancer following surgery was performed, and it was identified that the 5-year overall survival rate of patients with low expression of Rab1A protein was $75.31 \%$, while that of patients with high expression of Rab1A was only $48.61 \%$, as demonstrated in Fig. 4.

\section{Discussion}

Rab1A is a member of the Rab protein family, which is a family of at least 30 GTPases like Ras. The majority of the functions comprise the recognition and fusion processes of membrane vesicles, regulating the membrane transport process between cells $(21,25)$. A previous study has indicated that small

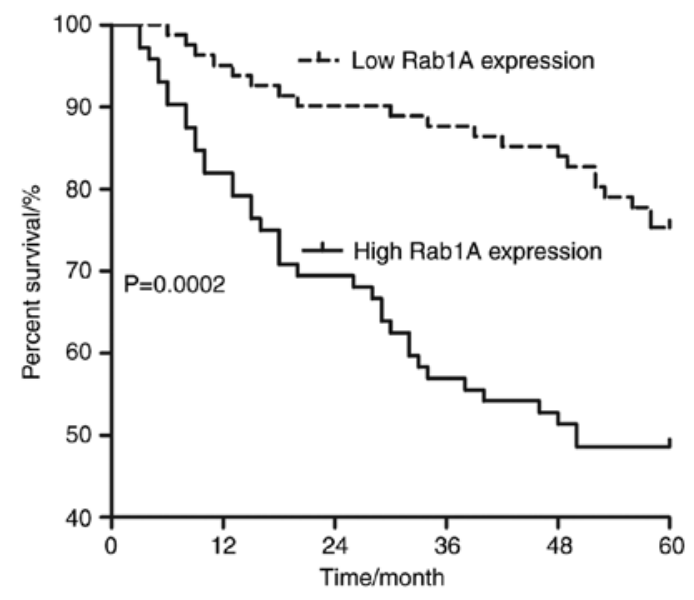

Figure 4. High expression of Rab1A protein decreases the 5-year survival rate in patients with bladder cancer. Rab1A, RAB1A, member RAS oncogene family.

molecule GTPases including the Ras, Rho, Rac and Ral families served important roles in tumorigenesis (26). In particular, Rab1A has been identified to be highly expressed in a variety of malignant tumor tissues as a small molecule GTPase and a member of the Ras oncogene superfamily $(20,21)$.

The present study identified that the expression levels of Rab1A protein in human bladder cancer cell lines were significantly increased compared with that in a human normal bladder epithelial cell line, and its expression level in human bladder cancer tissues was also significantly increased compared with that in adjacent normal tissues. In 2005, Rab1A was first protein identified to be highly expressed in tongue cancer (17); subsequently, the study of Rab1A in the field of malignant tumors has become more intense, and has demonstrated different degrees of increased expression of Rab1 A in various malignant tumors. It is widely hypothesized that its high expression promotes the development of cancer by regulating the mTOCRC1 signaling pathway $(27,28)$, which directly leads to poor prognoses in colorectal (21) and liver cancer (20). Combined with the results of the present study, these observations suggest that Rab1A protein, which was highly expressed in bladder cancer tissues, may be involved in the regulation of bladder cancer development.

Subsequent analysis performed in the present study revealed that Rab1A protein expression was significantly associated with histological grade, TNM stage and cancer metastasis in patients with bladder cancer, and that its expression was increased with later TNM stages. It was also demonstrated that Rab1A expression was significantly increased in patients with metastatic cancer compared with patients without metastasis. In addition, it was identified to be an independent risk factor affecting the prognosis of patients with bladder cancer. The occurrence and development of malignant tumors is a multi-step process in which multiple factors are combined through internal and external effects. The incidence of tumor cells transference from the primary site to other sites and the continuation of growth is not only a criterion for distinguishing between benign and malignant tumors, but also a major cause of treatment failure and mortality in patients with malignant tumors. The epithelial-mesenchymal transition process is a 
Table II. Cox univariate regression model analysis of survival of 153 patients with bladder cancer.

\begin{tabular}{|c|c|c|c|c|}
\hline Variable & $\mathrm{N}$ & $95 \% \mathrm{CI}$ & OR & P-value \\
\hline \multicolumn{5}{|l|}{ Sex } \\
\hline Female/male & $32 / 121$ & $1.056-4.512$ & 2.134 & 0.213 \\
\hline \multicolumn{5}{|l|}{ Age, years } \\
\hline$<60 / \geq 60$ & $62 / 91$ & $0.298-1.569$ & 0.935 & 0.328 \\
\hline \multicolumn{5}{|l|}{ Tumor size, cm } \\
\hline$<2.5 / \geq 2.5$ & $81 / 72$ & $0.650-2.634$ & 1.025 & 0.309 \\
\hline \multicolumn{5}{|l|}{ Histological grade } \\
\hline I/II-III & $72 / 81$ & $0.802-2.688$ & 1.596 & 0.022 \\
\hline \multicolumn{5}{|l|}{ Tumor number } \\
\hline Single/Multiple & $92 / 61$ & $0.782-3.697$ & 2.264 & 0.139 \\
\hline \multicolumn{5}{|l|}{ TNM } \\
\hline 0a-I/II-III & $85 / 61$ & $1.652-5.126$ & 9.105 & $<0.001$ \\
\hline \multicolumn{5}{|c|}{ Cancer cells metastasis } \\
\hline Yes/No & $31 / 122$ & $2.069-4.008$ & 3.269 & $<0.001$ \\
\hline \multicolumn{5}{|l|}{ Smoking } \\
\hline Yes/No & $103 / 50$ & $0.894-2.956$ & 1.027 & 0.042 \\
\hline \multicolumn{5}{|l|}{ Total cystectomy } \\
\hline Yes/No & $112 / 41$ & $1.023-4.781$ & 2.308 & 0.047 \\
\hline \multicolumn{5}{|l|}{ Rab1A expression } \\
\hline Low/High & $81 / 72$ & $1.229-5.224$ & 2.941 & 0.012 \\
\hline
\end{tabular}

Rab1A, RAB1A, member RAS oncogene family; TNM, tumor-node-metastasis; 96\% CI, 95\% confidence interval; OR, odds ratio.

Table III. Cox multivariate regression model analysis of survival of 153 patients with bladder cancer.

\begin{tabular}{lccr}
\hline Variables & $95 \%$ CI & OR & P-value \\
\hline Histological grade & $0.981-4.028$ & 2.021 & $<0.001$ \\
TNM & $1.154-4.218$ & 2.302 & $<0.001$ \\
Cancer cells metastasis & $1.102-8.648$ & 3.741 & 0.019 \\
Smoking & $0.549-3.694$ & 2.103 & 0.412 \\
Total cystectomy & $0.143-1.542$ & 0.512 & 0.089 \\
Rab1A expression & $0.139-0.916$ & 0.549 & 0.027 \\
\hline
\end{tabular}

Rab1A, RAB1A, member RAS oncogene family; TNM, tumornode-metastasis; 96\% CI, 95\% confidence interval; OR, odds ratio.

key step in conferring the ability of tumor cells to invade and migrate, and is one of the most important causes of mortality in patients with malignant tumors $(29,30)$. mTOR is an evolutionarily conserved serine/threonine protein kinase with two complexes, mTORC1 and mTORC2. Rab1A, a member of the Rag GTPase family, activates the mTORC1 signaling pathway. A number of previous studies have demonstrated that Rab1A may participate in tumor cell growth, apoptosis, invasion and migration by activating the mTORC1 signaling pathway: $\mathrm{Xu}$ et al (8) identified that in hepatocellular carcinoma, Rab1A was highly expressed and regulated the proliferation, growth, invasion and metastasis of hepatoma cells by activating the mTORC1 signaling pathway, and that the inhibition of Rab1A expression caused EMT inhibition. In addition, Yang et al (28) revealed that Rab1A and Rab1B, which were highly expressed in esophageal squamous cell carcinoma, inhibited autophagy in cancer cells and promoted cancer cell survival by activating the mTORC1 signaling pathway.

Previous studies have demonstrated that the loss or decreased expression of PTEN in the bladder mucosal epithelium that regulated the PI3K/Akt/mTOR signaling pathway led to dysfunction of this signaling pathway, causing the bladder cell epithelium to lose its normal cell cycle regulation and hyperproliferation and malignant transformation (31). Becker et al (32) identified that the combination of a mTORc1/TORc2 inhibitor and lapatinib inhibited the proliferation, invasion and migration of bladder cancer cells in vitro. These results suggested that Rab1A may participate in the development of bladder cancer by regulating the mTORC1 signaling pathway.

In conclusion, the expression of RablA in bladder cancer was upregulated, and its expression increased with increasing TNM stage. It is associated with the metastasis of tumor cells and negatively affects the survival time of patients with bladder cancer, and may be a potential target for the treatment of bladder cancer. However, the function of Rab1A at the cellular and whole organism level was not examined in bladder cancer. In order to improve the understanding of the function of Rab1A in bladder cancer, future studies are required. 


\section{Acknowledgements}

Not applicable.

\section{Funding}

No funding was received.

\section{Availability of data and materials}

All data generated or analyzed during this study are included in this published article.

\section{Authors' contributions}

HS made substantial contributions to the conception and design, acquisition of data and revising the critically for important intellectual content of this study. TL, XL, HL, CL and XDL analyzed and interpreted the data. All authors read and approved the final manuscript.

\section{Ethics approval and consent to participate}

All subjects (or their guardians) included in the present study consented to the study protocol and provided written informed consent. The Ethics Committee of The First Affiliated Hospital of Hebei North University approved the study.

\section{Patient consent for publication}

Not applicable.

\section{Competing interests}

The authors declare that they have no competing interests.

\section{References}

1. Siegel R, Naishadham D and Jemal A: Cancer statistics, 2012. Ca Cancer J Clin 62: 10-29, 2012.

2. Chen W, Zheng R, Baade PD, Zhang S, Zeng H, Bray F, Jemal A Yu XQ and He J: Cancer statistics in China, 2015. CA Cancer J Clin 66: 115-132, 2016.

3. Wei F, Wu Y, Tang L, Xiong F, Guo C, Li X, Zhou M, Xiang B, $\mathrm{LiX}, \mathrm{Li} \mathrm{G}$, et al: Trend analysis of cancer incidence and mortality in China. Sci China Life Sci 60: 1271-1275, 2017.

4. Quan Y, Song Q, Wang J, Zhao L, Lv J and Gong S: MiR-1202 functions as a tumor suppressor in glioma cells by targeting Rab1A. Tumor Biol 39: 101042831769756, 2017.

5. Allan BB, Moyer BD and Balch WE: Rab1 recruitment of p115 into a cis-SNARE complex: Programming budding COPII vesicles for fusion. Science 289: 444-448, 2000

6. Satoh A, Wang Y, Malsam J, Beard MB and Warren G: Golgin-84 is a rab1 binding partner involved in golgi structure. Traffic 4: $153-161,2010$.

7. Thomas JD, Zhang YJ, Wei YH, Cho JH, Morris LE, Wang HY and Zheng XF: Rab1A is an mTORC1 activator and a colorectal oncogene. Cancer Cell 26: 754-769, 2014.

8. Xu BH, Li XX, Wang HY and Zheng XS: Overexpressed Rab1A is associated poor prognosis and promotes oncogenic growth and metastasis through mTORC1 activation in hepatocellular carcinoma. AACR, 2015

9. Mukhopadhyay A, Nieves E, Che FY, Wang J, Jin L, Murray JW, Gordon K, Angeletti RH and Wolkoff AW: Proteomic analysis of endocytic vesicles: Rabla regulates motility of early endocytic vesicles. J Cell Sci 124: 765-775, 2011.
10. Mukhopadhyay A, Quiroz JA and Wolkoff AW: Rabla regulates sorting of early endocytic vesicles. Am J Physiol Gastrointest Liver Physiol 306: G412-G424, 2014.

11. Charng WL, Yamamoto S, Jaiswal M, Bayat V, Xiong B, Zhang K, Sandoval H, David G, Gibbs S, Lu HC, et al: Drosophila tempura, a novel protein prenyltransferase $\alpha$ subunit, regulates notch signaling Via Rab1 and Rab11. PLoS Biol 12: e1001777, 2014.

12. Wang C, Yoo Y, Fan H, Kim E, Guan KL and Guan JL: Regulation of Integrin $\beta 1$ recycling to lipid rafts by Rabla to promote cell migration. J Biol Chem 285: 29398-29405, 2010.

13. Tanaka M, Mun S, Harada A, Ohkawa Y, Inagaki A, Sano S, Takahashi K, Izumi Y, Osada-Oka M, Wanibuchi H, et al: Hsc70 contributes to cancer cell survival by preventing Rab1 A degradation under stress conditions. PLoS One 9: e96785, 2014.

14. Salem A, Almahmoudi R, Listyarifah D, Siponen M, Maaninka K, Al-Samadi A, Salo T and Eklund KK: Histamine $\mathrm{H}_{4}$ receptor signalling in tongue cancer and its potential role in oral carcinogenesis-a short report. Cell Oncol (Dordr) 40: 621-630, 2017.

15. Coune PG, Bensadoun JC, Aebischer P and Schneider BL: Rab1A over-expression prevents Golgi apparatus fragmentation and partially corrects motor deficits in an alpha-synuclein based rat model of Parkinson's disease. J Parkinsons Dis 1: 373-387, 2011.

16. Wu G, Yussman MG, Barrett TJ, Hahn HS, Osinska H, Hilliard GM, Wang X, Toyokawa T, Yatani A, Lynch RA, et al: Increased myocardial Rab GTPase expression: A consequence and cause of cardiomyopathy. Circ Res 89: 1130-1137, 2001.

17. Shimada K, Uzawa K, Kato M, Endo Y, Shiiba M, Bukawa H, Yokoe H, Seki N and Tanzawa H: Aberrant expression of RAB1A in human tongue cancer. Br J Cancer 92: 1915-1921, 2005.

18. Xu H, Qian M, Zhao B, Wu C, Maskey N, Song H, Li D, Song J, Hua K and Fang L: Inhibition of RAB1A suppresses epithelial-mesenchymal transition and proliferation of triple-negative breast cancer cells. Oncol Rep 37: 1619-1626, 2017.

19. Wang X, Liu F, Qin X, Huang T, Huang B, Zhang Y and Jiang B: Expression of Rab1A is upregulated in human lung cancer and associated with tumor size and T stage. Aging (Albany NY) 8: 2790-2798, 2016

20. Xu BH, Li XX, Yang Y, Zhang MY, Rao HL, Wang HY and Zheng XF: Aberrant amino acid signaling promotes growth and metastasis of hepatocellular carcinomas through Rab1A-dependent activation of mTORC1 by Rab1A. Oncotarget 6: 20813-20828, 2015.

21. Thomas JD, Zhang YJ, Wei YH, Cho JH, Morris LE, Wang HY and Zheng XFS: Rab1 A Is an mTORC1 activator and a colorectal oncogene. Cancer Cell 26: 181-182, 2016.

22. Fan SJ, Snell C, Turley H, Li JL, McCormick R, Perera SM, Heublein S, Kazi S, Azad A, Wilson C, et al: PAT4 levels control amino-acid sensitivity of rapamycin-resistant mTORC1 from the Golgi and affect clinical outcome in colorectal cancer. Oncogene 35: 3004-3015, 2016.

23. Kirkali Z, Chan T, Manoharan M, Algaba F, Busch C, Cheng L, Kiemeney L, Kriegmair M, Montironi R, Murphy WM, et al: Bladder cancer: Epidemiology, staging and grading, and diagnosis. Urology 66 (6 Suppl 1): S4-S34, 2005.

24. Herr HW: Pathologic evaluation of radical cystectomy specimens. Cancer 95: 668-669, 2002.

25. Hutagalung AH and Novick PJ: Role of Rab GTPases in membrane traffic and cell physiology. Physiol Rev 91: 119-149, 2011.

26. Li Y, Wang HY and Zheng XF: Rab1 GTPases as oncogenes. Aging (Albany NY) 7: 897-898, 2015.

27. RAB1A Promotes Oncogenesis in Colorectal Cancer via mTORC1 Activation. Cancer Discovery 4: 1366-1366, 2014.

28. Yang XZ, Wang R, Li XX, Yang Y, Wang HY and Zheng XS: Rab1A and Rab1B promote esophageal squamous cell carcinoma through activating mTORC1 signaling and inhibiting autophagy. AACR, 2016.

29. Zavadil J, Haley J, Kalluri R, Muthuswamy SK and Thompson E: Epithelial-mesenchymal transition. Cancer Res 68: 9574-9577, 2008.

30. Seton-Rogers S: Epithelial-mesenchymal transition: Untangling EMT's functions. Nat Rev Cancer 16: 1, 2016.

31. Hay N and Sonenberg N: Upstream and downstream of mTOR. Genes Dev 18: 1926-1945, 2004.

32. Becker MN, Wu KJ, Marlow LA, Kreinest PA, Vonroemeling CA, Copland JA and Williams CR: The combination of an mTORc1/TORc2 inhibitor with lapatinib is synergistic in bladder cancer in vitro. Urol Oncol 32: 317-326, 2014.

This work is licensed under a Creative Commons Attribution-NonCommercial-NoDerivatives 4.0 International (CC BY-NC-ND 4.0) License. 